\title{
Wilker-type inequalities for hyperbolic Fibonacci functions
}

\section{Mustafa Bahşi*}

${ }^{\text {*Correspondence: }}$ mhvbahsi@yahoo.com

Education Faculty, Aksaray

University, Aksaray, Turkey

\begin{abstract}
This article introduces the analog of the Wilker inequality and the parameterized Wilker inequality for the hyperbolic Fibonacci functions.
\end{abstract}

Keywords: Wilker's inequality; Wilker-Anglesio inequality; hyperbolic Fibonacci functions

\section{Introduction}

The inequalities

$$
\left(\frac{\sin x}{x}\right)^{2}+\frac{\tan x}{x}>2
$$

and

$$
\left(\frac{\sin x}{x}\right)^{2}+\frac{\tan x}{x}>2+c x^{3} \tan x
$$

were proposed first by Wilker [1] as open problems, where $0<x<\frac{\pi}{2}$ and $c$ is constant. The inequality (1) was proved by some authors [2-5]. In [2], Anglesio showed that the sharp inequality

$$
\left(\frac{\sin x}{x}\right)^{2}+\frac{\tan x}{x}>2+\frac{16}{\pi^{4}} x^{3} \tan x
$$

is valid and the best possible constant is $\frac{16}{\pi^{4}}$ for the constant $c$ in inequality (2). Also, the following similar inequality to inequality (1) was proved by Huygens [6]:

$$
2\left(\frac{\sin x}{x}\right)+\frac{\tan x}{x}>3
$$

During the past few years, some inequalities have appeared as the generalizations and improvements of the Wilker inequality (1) and the Wilker-Anglesio inequality (3) [7-11]. In [11], Wu and Srivastava gave a generalization of Wilker's inequality involving parameters of exponent and weight, as follows:

$$
\frac{\lambda}{\lambda+\mu}\left(\frac{\sin x}{x}\right)^{p}+\frac{\mu}{\lambda+\mu}\left(\frac{\tan x}{x}\right)^{q}>1,
$$

(c) 2016 Bahşi. This article is distributed under the terms of the Creative Commons Attribution 4.0 International License (http://creativecommons.org/licenses/by/4.0/), which permits unrestricted use, distribution, and reproduction in any medium, provided you give appropriate credit to the original author(s) and the source, provide a link to the Creative Commons license, and indicate if changes were made. 
where $0<x<\frac{\pi}{2}, \lambda>0, \mu>0, p \leq \frac{2 q \mu}{\lambda}, q>0$ or $q \leq \min \left\{-\frac{\lambda}{\mu},-1\right\}$. Moreover, Wilker and Anglesio type inequalities have been obtained for the hyperbolic functions [12, 13]. Wu and Debnath [12] established the hyperbolic analog of the Wilker-Anglesio inequality and the parameterized Wilker inequality as follows:

$$
\begin{aligned}
& \left(\frac{\sinh x}{x}\right)^{2}+\frac{\tanh x}{x}>2+\frac{8}{45} x^{3} \tanh x, \\
& \frac{\lambda}{\lambda+\mu}\left(\frac{\sinh x}{x}\right)^{p}+\frac{\mu}{\lambda+\mu}\left(\frac{\tanh x}{x}\right)^{q}>1,
\end{aligned}
$$

where $x \neq 0, \lambda>0, \mu>0, p \geq \frac{2 q \mu}{\lambda}$, and $q>0$ or $q \leq \min \left\{-\frac{\lambda}{\mu},-1\right\}$.

The aim of this paper is to present the analog of the Wilker-Anglesio inequality (6) and the parameterized Wilker inequality (7) together with some applications for the hyperbolic Fibonacci functions.

\section{Preliminaries and some lemmas}

The Fibonacci numbers are defined by the second order linear recurrence relation: $F_{n+1}=$ $F_{n}+F_{n-1}(n \geq 1)$ with the initial conditions $F_{0}=0$ and $F_{1}=1$. Similarly, the Lucas numbers are defined by $L_{n+1}=L_{n}+L_{n-1}(n \geq 1)$ with the initial conditions $L_{0}=2$ and $L_{1}=1$. The characteristic equation of $F_{n}$ is

$$
t^{2}-t-1=0
$$

The roots of equation (8) are $\alpha=\frac{1+\sqrt{5}}{2}, \beta=\frac{1-\sqrt{5}}{2}$, and the Binet formula for $F_{n}$ is

$$
F_{n}= \begin{cases}\frac{\alpha^{n}+\alpha^{-n}}{\sqrt{5}}, & n \text { odd } \\ \frac{\alpha^{n}-\alpha^{-n}}{\sqrt{5}}, & n \text { even } .\end{cases}
$$

The positive root of equation (8), $\alpha=\frac{1+\sqrt{5}}{2}$, is called the golden ratio, which has been very attractive for researchers because it occurs ubiquitous such as in nature, art, architecture, and anatomy.

The Fibonacci numbers have many properties, continuous versions, and generalizations [14-20]. Stakhov and Tkachenko [14] introduced a new class of hyperbolic functions called hyperbolic Fibonacci functions replacing the discrete variable $n$ in equation (9) with the continuous variable $x$ that takes its values from the set of real numbers. Based on an analogy between the Binet formula, (9), and the classical hyperbolic functions,

$$
\sinh (x)=\frac{e^{x}-e^{-x}}{2} \quad \text { and } \quad \cosh (x)=\frac{e^{x}+e^{-x}}{2}
$$

Stakhov and Rozin [15] defined the so-called symmetrical hyperbolic Fibonacci functions as follows:

$$
s F s(x)=\frac{\alpha^{x}-\alpha^{-x}}{\sqrt{5}} \quad \text { and } \quad c F s(x)=\frac{\alpha^{x}+\alpha^{-x}}{\sqrt{5}},
$$

where $s F s(x)$ and $c F s(x)$ denote symmetric hyperbolic Fibonacci sine and cosine functions, respectively. Similarly, a symmetric hyperbolic Fibonacci tangent function can be defined 


$$
t F s(x)=\frac{s F s(x)}{c F s(x)}=\frac{\alpha^{x}-\alpha^{-x}}{\alpha^{x}+\alpha^{-x}}
$$

The graphs of the symmetrical hyperbolic Fibonacci functions have a symmetric form and are similar to the graphs of the classical hyperbolic functions. Also, the symmetrical hyperbolic Fibonacci functions $s F s(x)$ and $c F s(x)$ are increasing on $(0,+\infty)$. The graphs of the symmetrical hyperbolic Fibonacci functions are given in [15]. The symmetric hyperbolic Fibonacci functions have properties that are similar to the classical hyperbolic functions. Some of them are [15]:

$$
c F s(x)=c F s(-x), \quad s F s(x)=-s F s(-x) \quad \text { and } \quad[c F s(x)]^{2}-[s F s(x)]^{2}=\frac{4}{5}
$$

Also, the derivative hyperbolic Fibonacci functions are [15]

$$
\begin{aligned}
& {[c F s(x)]^{(n)}= \begin{cases}(\ln \alpha)^{n} s F s(x), & \text { for } n \text { odd, } \\
(\ln \alpha)^{n} c F s(x), & \text { for } n \text { even, }\end{cases} } \\
& {[s F s(x)]^{(n)}= \begin{cases}(\ln \alpha)^{n} c F s(x), & \text { for } n \text { odd, } \\
(\ln \alpha)^{n} s F s(x), & \text { for } n \text { even. }\end{cases} }
\end{aligned}
$$

For more information and the generalizations as regards hyperbolic Fibonacci functions, see [15-20] the references cited therein.

Throughout this paper $s F s(x), c F s(x)$, and $t F s(x)$ denote the hyperbolic Fibonacci functions given in (11) and (12) and $\alpha$ denotes the golden ratio, $\alpha=\frac{1+\sqrt{5}}{2}$.

Lemma 1 [21] If $x_{i}>0, \lambda_{i}>0(i=1,2, \ldots, n)$, and $\sum_{i=1}^{n} \lambda_{i}=1$, then

$$
\sum_{i=1}^{n} \lambda_{i} x_{i} \geq \prod_{i=1}^{n} x_{i}^{\lambda_{i}}
$$

Lemma 2 For all nonzero real numbers $x$, the following inequality holds:

$$
\frac{2}{\sqrt{5}} \leq c F s(x) \leq \frac{5}{4(\ln (\alpha))^{3}}\left(\frac{s F s(x)}{x}\right)^{3}
$$

Proof From $c F s(0)=\frac{2}{\sqrt{5}}, c F s(x)=c F s(-x)$, and $c F s(x)$ is increasing on $(0,+\infty)$, the left hand side inequality of (13) is true. Let us prove the right hand side inequality of (13).

Case (I): For $x>0$, define a function $f: \mathbb{R}^{+} \rightarrow \mathbb{R}$ by

$$
f(x)=\frac{s F s^{3}(x)}{x^{3} c F s(x)}
$$

By differentiating with respect to $x$, we get

$$
f^{\prime}(x)=\frac{s F s^{2}(x)}{x^{4} c F s^{2}(x)}\left[2 x \ln (\alpha) s F s^{2}(x)+\frac{12}{5} x \ln (\alpha)-3 s F s(x) c F s(x)\right]=\frac{s F s^{2}(x)}{x^{4} c F s^{2}(x)} f_{1}(x)
$$




$$
\begin{aligned}
& f_{1}^{\prime}(x)=4 \ln (\alpha) s F s(x) c F s(x)\left[x \ln (\alpha)-\frac{s F s(x)}{c F s(x)}\right]=4 \ln (\alpha) s F s(x) c F s(x) f_{2}(x), \\
& f_{2}^{\prime}(x)=\ln (\alpha)\left(\frac{s F s(x)}{c F s(x)}\right)^{2}>0 .
\end{aligned}
$$

This means that $f_{2}(x)$ is increasing on $(0,+\infty)$. We thus conclude from $f_{2}(0)=f_{1}(0)=0$ that $f_{2}(x)>0$ and $f_{1}(x)$ is increasing and positive on $(0,+\infty)$. Hence, we see that $f(x)$ is increasing on $(0,+\infty)$. By using

$$
\lim _{x \rightarrow 0^{+}} f(x)=\frac{4(\ln (\alpha))^{3}}{5},
$$

we conclude that

$$
c F s(x)<\frac{5}{4(\ln (\alpha))^{3}}\left(\frac{s F s(x)}{x}\right)^{3} .
$$

Case (II): Let $x<0(-x>0)$. Since $c F s(-x)=c F s(x)$ and $s F s(-x)=-s F s(x)$ and considering the result proved in Case (I), the proof is completed.

\section{Wilker-Anglesio's inequality for hyperbolic Fibonacci functions}

Theorem 1 Let $x$ be nonzero real numbers; then the following inequality holds:

$$
\left(\frac{s F s(x)}{x}\right)^{2}+\frac{t F s(x)}{x}>\frac{8(\ln (\alpha))^{2}}{5}+\frac{32(\ln (\alpha))^{5} x^{3}}{225} t F s(x) .
$$

Proof From $s F s(-x)=-s F s(x)$ and $t F s(-x)=-t F s(x)$, we have

$$
\left(\frac{s F s(-x)}{(-x)}\right)^{2}+\frac{t F s(-x)}{(-x)}=\left(\frac{s F s(x)}{x}\right)^{2}+\frac{t F s(x)}{x} .
$$

Thus, it is enough to prove that Theorem 1 is true for $x>0$. For this purpose, define a function $g: \mathbb{R}^{+} \rightarrow \mathbb{R}$ by

$$
g(x)=\frac{\frac{5}{4(\ln (\alpha))^{2}}\left(\frac{s F s(x)}{x}\right)^{2}+\frac{1}{\ln (\alpha)} \frac{t F s(x)}{x}-2}{x^{3} t F s(x)} .
$$

Then, upon differentiating with respect to $x$, we get

$$
\begin{aligned}
g^{\prime}(x)= & \frac{1}{4 \sqrt{5} \ln (\alpha) x^{6} s F s^{2}(x)}\left[2 x c F s(4 x)+24 x^{2} \ln (\alpha) s F s(2 x)-\frac{5}{\ln (\alpha)} s F s(4 x)\right. \\
& \left.+\frac{10}{\ln (\alpha)} s F s(2 x)-20 x c F s(2 x)+\left(40-\frac{4 \sqrt{5}}{5}\right) x+\frac{2 \sqrt{5}}{5}(\ln (\alpha))^{2} 16 x^{3}\right] \\
= & \frac{g_{1}(x)}{4 \sqrt{5} \ln (\alpha) x^{6} s F s^{2}(x)} \\
g_{1}^{\prime}(x)= & 8 \sqrt{5} c F s^{2}(x)\left[6 x^{2}(\ln (\alpha))^{2}+x \ln (\alpha) s F s(x)\left[5 c F s(x)-\frac{1}{c F s(x)}\right]-\frac{90}{8} s F s^{2}(x)\right] \\
= & 8 \sqrt{5} c F s^{2}(x) g_{2}(x),
\end{aligned}
$$




$$
\begin{aligned}
g_{2}^{\prime}(x)= & \frac{1}{c F s^{2}(x)}\left[-\frac{35}{2} \ln (\alpha) s F s(x) c F s^{3}(x)-\ln (\alpha) s F s(x) c F s(x)\right. \\
& \left.+10 x(\ln (\alpha))^{2} c F s^{4}(x)+8 x(\ln (\alpha))^{2} c F s^{2}(x)-\frac{4}{5} x(\ln (\alpha))^{2}\right]=\frac{g_{3}(x)}{c F s^{2}(x)} \\
g_{3}^{\prime}(x)= & 4(\ln (\alpha))^{2} s F s(2 x)\left[\frac{8}{\sqrt{5}} x \ln (\alpha)-3 s F s(2 x)+2 x \ln (\alpha) c F s(2 x)\right] \\
= & 4(\ln (\alpha))^{2} s F s(2 x) g_{4}(x) \\
g_{4}^{\prime}(x)= & 4 \ln (\alpha) s F s(2 x)\left[x \ln (\alpha)-\frac{s F s(x)}{c F s(x)}\right] \\
= & 4 \ln (\alpha) s F s(2 x) g_{5}(x) \\
g_{5}^{\prime}(x)= & \ln (\alpha)\left(\frac{s F s(x)}{c F s(x)}\right)^{2}>0 .
\end{aligned}
$$

This means that $g_{5}(x)$ is increasing on the open interval $(0,+\infty)$. By $g_{5}(0)=g_{4}(0)=g_{3}(0)=$ $g_{2}(0)=g_{1}(0)=0$ this immediately shows that $g_{5}(x), g_{4}(x), g_{3}(x), g_{2}(x)$, and $g_{1}(x)$ are increasing and positive on $(0,+\infty)$. Thus, we see that $g(x)$ is increasing on $(0,+\infty)$. Also, we use

$$
\lim _{x \rightarrow 0^{+}} g(x)=\frac{8(\ln (\alpha))^{3}}{45}
$$

Hence, we conclude from

$$
\frac{\frac{5}{4(\ln (\alpha))^{2}}\left[\left(\frac{s F s(x)}{x}\right)^{2}+\frac{t F s(x)}{x}\right]-2}{x^{3} t F s(x)}>\frac{\frac{5}{4(\ln (\alpha))^{2}}\left(\frac{s F s(x)}{x}\right)^{2}+\frac{1}{\ln (\alpha)} \frac{t F s(x)}{x}-2}{x^{3} t F s(x)}
$$

that

$$
\left(\frac{s F s(x)}{x}\right)^{2}+\frac{t F s(x)}{x}>\frac{8(\ln (\alpha))^{2}}{5}+\frac{32(\ln (\alpha))^{5} x^{3}}{225} t F s(x) .
$$

This completes the proof.

Next we give parameterized Wilker's inequality for hyperbolic Fibonacci functions.

Theorem 2 For the hyperbolic Fibonaccifunctions, the following inequality holds:

$$
\frac{\lambda}{\lambda+\mu}\left(\frac{s F s(x)}{x}\right)^{p}+\frac{\mu}{\lambda+\mu}\left(\frac{t F s(x)}{x}\right)^{q}>\left(\frac{2 \ln (\alpha)}{\sqrt{5}}\right)^{\frac{p \lambda+q \mu}{\lambda+\mu}},
$$

where $x \neq 0, \lambda>0, \mu>0, p \geq \frac{2 q \mu}{\lambda}$, and $q>0$.

Proof From Lemmas 1 and 2, we have

$$
\begin{gathered}
\frac{\lambda}{\lambda+\mu}\left(\frac{s F s(x)}{x}\right)^{p}+\frac{\mu}{\lambda+\mu}\left(\frac{t F s(x)}{x}\right)^{q} \\
\geq\left(\frac{s F s(x)}{x}\right)^{\frac{p \lambda}{\lambda+\mu}}\left(\frac{t F s(x)}{x}\right)^{\frac{q \mu}{\lambda+\mu}}
\end{gathered}
$$




$$
\begin{aligned}
& =\left(\frac{s F s(x)}{x}\right)^{\frac{p \lambda}{\lambda+\mu}}\left(\frac{s F s(x)}{x}\right)^{\frac{q \mu}{\lambda+\mu}}\left(\frac{1}{c F s(x)}\right)^{\frac{q \mu}{\lambda+\mu}} \\
& >\left(\frac{s F s(x)}{x}\right)^{\frac{p \lambda+q \mu}{\lambda+\mu}}\left(\frac{s F s(x)}{x}\right)^{\frac{-3 q \mu}{\lambda+\mu}}\left(\frac{5}{4(\ln (\alpha))^{3}}\right)^{\frac{-q \mu}{\lambda+\mu}} \\
& =\left(\frac{s F s(x)}{x}\right)^{\frac{p \lambda-2 q \mu}{\lambda+\mu}}\left(\frac{5}{4(\ln (\alpha))^{3}}\right)^{\frac{-q \mu}{\lambda+\mu}} \\
& >\left(\frac{2 \ln (\alpha)}{\sqrt{5}}\right)^{\frac{p \lambda-2 q \mu}{\lambda+\mu}}\left(\frac{4(\ln (\alpha))^{3}}{5}\right)^{\frac{q \mu}{\lambda+\mu}} \\
& >\left(\frac{2 \ln (\alpha)}{\sqrt{5}}\right)^{\frac{p \lambda+q \mu}{\lambda+\mu}} .
\end{aligned}
$$

Now we give some weighted and exponential Wilker-type inequalities for hyperbolic Fibonacci functions as applications of Theorem 2.

Corollary 1 Let $x \neq 0, \lambda \geq \mu>0$ and $(p, q)=(2,1)$. Then

$$
\frac{\lambda}{\lambda+\mu}\left(\frac{s F s(x)}{x}\right)^{2}+\frac{\mu}{\lambda+\mu}\left(\frac{t F s(x)}{x}\right)>\frac{4(\ln (\alpha))^{2}}{5} .
$$

Corollary 2 Let $x \neq 0, p \geq q>0$, and $(\lambda, \mu)=(2,1)$. Then

$$
2\left(\frac{s F s(x)}{x}\right)^{p}+\left(\frac{t F s(x)}{x}\right)^{q}>3\left(\frac{2 \ln (\alpha)}{\sqrt{5}}\right)^{p}
$$

It is obvious that the inequality (14) reduces to Huygens type inequality for $p=q=1$.

\section{Competing interests}

The author declares that he has no competing interests.

\section{Acknowledgements}

The author is grateful to the referees and the associate editor for their careful reading, helpful comments, and constructive suggestions, which improved the presentation of the results.

Received: 23 November 2015 Accepted: 19 May 2016 Published online: 02 June 2016

\section{References}

1. Wilker, JB: Problem E3306. Am. Math. Mon. 96, 55 (1989)

2. Sumner, JS, Jagers, AA, Vowe, M, Anglesio, J: Inequalities involving trigonometric functions. Am. Math. Mon. 98, 264-267 (1991)

3. Guo, B-N, Li, W, Qi, F: Proofs of Wilker's inequalities involving trigonometric functions. In: Inequality Theory and Applications. Chinju/Masan, 2001, vol. 2, pp. 109-112. Nova Science Publishers, New York (2003)

4. Zhang, L, Zhu, L: A new elementary proof of Wilker's inequalities. Math. Inequal. Appl. 11(1), 149-151 (2008)

5. Zhu, L: A new simple proof of Wilker's inequality. Math. Inequal. Appl. 8(4), 749-750 (2005)

6. Huygens, C: Oeuvres Completes. Société Hollondaise des Science, Haga (1888-1940)

7. Zhu, L: On Wilker-type inequalities. Math. Inequal. Appl. 10(4), 727-731 (2007)

8. Guo, B-N, Qiao, B-M, Qi, F, Li, W: On new proofs of Wilker's inequalities involving trigonometric functions. Math. Inequal. Appl. 6(1), 19-22 (2003)

9. Pinelis, l: L'Hospital rules for monotonicity and the Wilker-Anglesio inequality. Am. Math. Mon. 111, 905-909 (2004)

10. Zhu, L: Inequalities for hyperbolic functions and their applications. J. Inequal. Appl. 2010, Article ID 130821 (2010). doi:10.1155/2010/130821

11. Wu, S-H, Srivastava, HM: A weighted and exponential generalization of Wilker's inequality and its applications. Integral Transforms Spec. Funct. 18(8), 529-535 (2007)

12. Wu, S-H, Debnath, L: Wilker-type inequalities for hyperbolic functions. Appl. Math. Lett. 25(5), 837-842 (2012)

13. Neuman, E: Wilker and Huygens-type inequalities for the generalized trigonometric and for the generalized hyperbolic functions. Appl. Math. Comput. 230, 211-217 (2014)

14. Stakhov, AP, Tkachenko, IS: Hyperbolic Fibonacci trigonometry. Rep Ukr. Acad. Sci. 7, 9-14 (1993) 
15. Stakhov, A, Rozin, B: On a new class of hyperbolic functions. Chaos Solitons Fractals 23(2), 379-389 (2005)

16. Stakhov, A, Rozin, B: The golden shofar. Chaos Solitons Fractals 26(3), 677-684 (2005)

17. Stakhov, A, Rozin, B: The "golden" hyperbolic models of Universe. Chaos Solitons Fractals 34(2), 159-171 (2007)

18. Falcón, S, Plaza, A: The k-Fibonacci hyperbolic functions. Chaos Solitons Fractals 38, 409-420 (2008)

19. Koçer, EG, Tuğlu, N, Stakhov, A: Hyperbolic functions with second order recurrence sequences. Ars Comb. 88, 65-81 (2008)

20. Vajda, S: Fibonacci and Lucas Numbers, and the Golden Section: Theory and Applications. Ellis Horwood, Chichester (1989)

21. Hardy, GH, Littlewood, JE, Pólya, G: Inequalities, 2nd edn. Cambridge University Press, Cambridge (1952)

Submit your manuscript to a SpringerOpen ${ }^{\circ}$ journal and benefit from:

- Convenient online submission

Rigorous peer review

- Immediate publication on acceptance

- Open access: articles freely available online

- High visibility within the field

- Retaining the copyright to your article 